\title{
Corrigendum: Supplementary Effect of Choline Alfoscerate on Speech Recognition in Patients With Age-Related Hearing Loss: A Prospective Study in 34 Patients (57 Ears)
}

\author{
Gina $\mathrm{Na}^{1,2}$, Sang Hyun Kwak ${ }^{3}$, Seung Hyun Jang ${ }^{1}$, Hye Eun Noh ${ }^{1}$, Jungghi Kim ${ }^{1}$, \\ SeungJoon Yang $^{1}$ and Jinsei Jung ${ }^{1 *}$ \\ ${ }^{1}$ Department of Otorhinolaryngology, Yonsei University College of Medicine, Seoul, South Korea, ${ }^{2}$ Department of \\ Otorhinolaryngology, Ilsan Paik Hospital, Inje University College of Medicine, Goyang, South Korea, ${ }^{3}$ Department of \\ Otorhinolaryngology, St. Vincent Hospital, College of Medicine, The Catholic University of Korea, Seoul, South Korea
}

Keywords: age-related hearing loss, hearing aids, choline alfoscerate, abbreviated profile of hearing aid benefit, listening comprehension

\section{A Corrigendum on}

OPEN ACCESS

Approved by:

Frontiers Editorial Office,

Frontiers Media SA, Switzerland

${ }^{*}$ Correspondence:

Jinsei Jung

jsjung@yuhs.ac

Received: 25 June 2021 Accepted: 28 June 2021

Published: 23 July 2021

Citation:

Na G, Kwak SH, Jang SH, Noh HE,

Kim J, Yang S and Jung J (2021)

Corrigendum: Supplementary Effect of

Choline Alfoscerate on Speech

Recognition in Patients With

Age-Related Hearing Loss: A

Prospective Study in 34 Patients

(57 Ears).

Front. Aging Neurosci. 13:730527.

doi: 10.3389/fnagi.2021.730527
Supplementary Effect of Choline Alfoscerate on Speech Recognition in Patients With Age-Related Hearing Loss: A Prospective Study in 34 Patients (57 Ears)

by Na, G., Kwak, S. H., Jang, S. H., Noh, H. E., Kim, J., Yang, et al. (2021). Front. Aging Neurosci. 13:684519. doi: 10.3389/fnagi.2021.684519

In the original article, there was an error in the Funding statement. The correct number for the National Research Foundation of Korea (NRF), funded by the Ministry of Science, ICT \& Future Planning is NRF-2019R1A2C1084033. The corrected Funding statement is shown below.

This work was supported by the National Research Foundation of Korea (NRF), funded by the Ministry of Science, ICT \& Future Planning (grant number NRF-2019R1A2C1084033 to JJ).

The authors apologize for this error and state that this does not change the scientific conclusions of the article in any way. The original article has been updated.

Publisher's Note: All claims expressed in this article are solely those of the authors and do not necessarily represent those of their affiliated organizations, or those of the publisher, the editors and the reviewers. Any product that may be evaluated in this article, or claim that may be made by its manufacturer, is not guaranteed or endorsed by the publisher.

Copyright (๑) 2021 Na, Kwak, Jang, Noh, Kim, Yang and Jung. This is an open-access article distributed under the terms of the Creative Commons Attribution License (CC BY). The use, distribution or reproduction in other forums is permitted, provided the original author(s) and the copyright owner(s) are credited and that the original publication in this journal is cited, in accordance with accepted academic practice. No use, distribution or reproduction is permitted which does not comply with these terms. 